\title{
Chronic exposure to simulated space conditions predominantly affects cytoskeleton remodeling and oxidative stress response in mouse fetal fibroblasts
}

\author{
MICHAËL BECK ${ }^{1,2}$, MARJAN MOREELS ${ }^{1}$, ROEL QUINTENS ${ }^{1}$, KHALIL ABOU-EL-ARDAT ${ }^{1,2}$, \\ HUSSEIN EL-SAGHIRE ${ }^{1,3}$, KEVIN TABURY ${ }^{1}$, ARLETTE MICHAUX ${ }^{1}$, ANN JANSSEN ${ }^{1}$, MIEKE NEEFS ${ }^{1}$, \\ PATRICK VAN OOSTVELDT ${ }^{2,4}$, WINNOK H. DE VOS ${ }^{2,4,5}$ and SARAH BAATOUT ${ }^{1,2}$

\begin{abstract}
${ }^{1}$ Radiobiology Unit, Expert Group of Molecular and Cellular Biology, Institute for Environment, Health and Safety, Belgian Nuclear Research Centre (SCK-CEN), Mol; ${ }^{2}$ Department of Molecular Biotechnology, ${ }^{3}$ Department of Basic Medical Sciences, Faculty of Medicine and Health Sciences, ${ }^{4}$ NB-Photonics, Ghent University, Ghent;

${ }^{5}$ Laboratory of Cell Biology and Histology, Department of Veterinary Sciences, Antwerp University, Antwerp, Belgium
\end{abstract}

Received February 2, 2014; Accepted March 24, 2014

DOI: $10.3892 / \mathrm{ijmm} .2014 .1785$

\begin{abstract}
Microgravity and cosmic rays as found in space are difficult to recreate on earth. However, ground-based models exist to simulate space flight experiments. In the present study, an experimental model was utilized to monitor gene expression changes in fetal skin fibroblasts of murine origin. Cells were continuously subjected for $65 \mathrm{~h}$ to a low dose $(55 \mathrm{mSv})$ of ionizing radiation (IR), comprising a mixture of high-linear energy transfer (LET) neutrons and low-LET gamma-rays, and/or simulated microgravity using the random positioning machine (RPM), after which microarrays were performed. The data were analyzed both by gene set enrichment analysis (GSEA) and single gene analysis (SGA). Simulated microgravity affected fetal murine fibroblasts by inducing oxidative stress responsive genes. Three of these genes are targets of the nuclear factor-erythroid 2 p45-related factor 2 (Nrf2), which may play a role in the cell response to simulated microgravity. In addition, simulated gravity decreased the expression of genes involved in cytoskeleton remodeling, which may have been caused by the downregulation of the serum response factor (SRF), possibly through the Rho signaling pathway. Similarly, chronic exposure to low-dose IR caused the downregulation of genes involved in cytoskeleton remodeling, as well as in cell cycle regulation and DNA damage response pathways. Many of
\end{abstract}

Correspondence to: Professor Sarah Baatout, Radiobiology Unit, Expert Group of Molecular and Cellular Biology, Institute for Environment, Health and Safety, Belgian Nuclear Research Centre (SCK-CEN), Boeretang 200, B-2400 Mol, Belgium

E-mail: sarah.baatout@sckcen.be

Key words: simulated space conditions, microarrays, cytoskeleton, oxidative stress, DNA damage the genes or gene sets that were altered in the individual treatments (RPM or IR) were not altered in the combined treatment (RPM and IR), indicating a complex interaction between RPM and IR.

\section{Introduction}

In the present study, we established an in vitro model in which primary cultures of fetal fibroblasts from murine origin (PFC) were subjected for $65 \mathrm{~h}$ to simulated microgravity, chronic irradiation or a combination. Genome-wide gene expression changes were thereafter assessed by microarrays. For microgravity simulation, we used the random positioning machine (RPM), which is one of the most widely used instruments for this purpose and has proven valuable in many cell types (1-6). As far as cosmic radiation is concerned, simulating the wide variety of ions ranging from low to very high energies encountered in space is problematic, particularly if irradiation is combined with microgravity simulation models. At present, no facility offers the possibility of producing chronic exposures of very high-energy beams consisting of multiple charged particles. We therefore used a source of californium Cf-252 for low-dose rate long-term exposure consisting of a mixture of high-linear energy transfer (LET) neutrons and low-LET gamma-rays (7).

The large amount of data generated with a highthroughput technology such as microarrays constitutes a double-edged sword: whole expression pattern may be recorded, but extracting the relevant information becomes more challenging $(8,9)$. To overcome this problem, analysis tools have been developed, such as single gene statistical analysis methods (SGA), which are widely used to determine the differentially expressed genes, and the gene set enrichment analysis (GSEA), which aims to identify gene expression differences in groups of genes, for instance in those acting synergistically in a cell process $(9,10)$. The two analytical methods were used concomitantly in this study. 


\section{Materials and methods}

Cell culture. All the animals were handled following the Belgian legislation after approval by the appropriate Ethics Committees (agreement number 08-002). BALB/cJ Rj (Janvier Laboratories, Saint-Berthevin, France) fetuses (three males and three females) originating from two different litters were dissected 17 days post-conception (day 0 being the fertilization day). Their skin was harvested and mechanically dissociated. The obtained tissue was enzymatically digested for $1 \mathrm{~h}$ at $37^{\circ} \mathrm{C}$ in phosphate-buffered saline (PBS; N.V. Invitrogen SA, Merelbeke, Belgium) solution containing $1 \mathrm{mg} / \mathrm{ml}$ of collagenase/dispase (Roche, Mannheim, Germany) and $5 \mathrm{mg} / \mathrm{ml}$ of trypsin 2,000 E/g (Merck KGaA, Darmstadt, Germany). The enzymatic reaction was subsequently stopped by adding fetal bovine serum (FBS; N.V. Invitrogen SA). The obtained cell suspension was subsequently centrifuged for $10 \mathrm{~min}$ at $350 \mathrm{xg}$ and the cells were seeded in 6-well plates in F12 medium supplemented with $20 \%$ FBS and $1 \%$ penicillin/streptomycin (both from N.V. Invitrogen SA), one fetus skin in each well. The cells were allowed to grow for up to 3 or 4 passages at $37^{\circ} \mathrm{C}\left(5 \%, \mathrm{CO}_{2}\right)$ and were subsequently frozen in FBS with $10 \%$ dimethyl sulfoxide (Sigma-Aldrich, St. Louis, MO, USA). The primary cultures were then thawed and allowed to grow for two weeks. The cells were seeded at a density of $\mathrm{x} 10^{5}$ cells in $12.5 \mathrm{~cm}^{2}$ flasks and allowed to adhere for $24 \mathrm{~h}$ prior to treatment.

Simulation of space conditions. Exposure to simulated space conditions included microgravity simulation using the desktop RPM (Dutch Space, Leiden, The Netherlands) and ionizing radiation (IR) (7). The exposure lasted for a period of $65 \mathrm{~h}$. Four treatment conditions were used: controls (CTRL), microgravity simulation (RPM), irradiation and a combination of the treatment methods (RPM and IR). For microgravity simulation, the flasks were completely filled with medium, sealed and placed on the RPM at a rotational velocity between 55 and $65^{\circ} / \mathrm{sec}$. Direction, speed and interval were set as random. The CTRL were placed in the same incubator under the same conditions as the treated samples. For chronic low-dose irradiation, the cells were exposed to a mixture of neutrons (98.2\%) and gamma-rays (1.8\%) directly or indirectly originating from a Cf-252 source were placed at $4.13 \mathrm{~m}$ from the incubator. The dosimetry was performed with bubble detectors as previously described (11) for neutron irradiation and with $600 \mathrm{cc}$ ionization chamber (NE) coupled with a Farmer electrometer for gamma-rays. The total dose received was $55.94 \pm 19.70 \mathrm{mSv}(862 \mu \mathrm{Sv} / \mathrm{h})$, which approximately corresponds to 35 times the dose rate measured on the International Space Station (ISS) (12), the total dose corresponding approximately to a stay of 100 days in the ISS.

RNA extraction. Immediately after treatment, adherent cells were washed in PBS, lysed in $350 \mathrm{ml}$ of AllPrep DNA/RNA/Protein Mini kit lysis buffer (Qiagen, Hilden, Germany) and frozen at $-80^{\circ} \mathrm{C}$. RNA was extracted using the same kit and its concentration was measured using the Nanodrop spectrophotometer (Thermo Scientific, Waltham, MA, USA) while its quality (RNA integrity number, RIN) was determined with Agilent's lab-on-chip Bioanalyzer 2100
(Agilent Technologies, Inc., Palo Alto, CA, USA). All the RNA samples had a RIN value of $>9.0$.

Affymetrixmicroarraysand dataanalysis. The RNA was treated using the GeneChip WT cDNA Synthesis and Amplification kit (Affymetrix, Santa Clara, CA, USA) according to the manufacturer's instructions. The resulting RNA was hybridized onto Affymetrix Mouse Gene 1.0 ST arrays.

Raw data (.cel-files) were imported at exon level in Partek Genomics Suite v6.5 (Partek Incorporated, St. Louis, MO, USA). Briefly, robust Multi-array Average (RMA) background correction was applied, data were normalized by quantile normalization and probe set summarization was performed using the median polish method. Gene summarization was performed using One-Step Tukey's Biweight method. These data were further analyzed with the Partek Genomics Suite software for SGA and by the GSEA software (v2.0, Broad Institute of Harvard and MIT, Cambridge, MA, USA).

For the single gene method, taking into consideration the scan date (also available for the litter), the fetus, the gender and the treatment as factors, a four-way ANOVA was performed to determine the genes that had a significantly altered expression for different conditions. For the pathway analysis, KEGG and PathArt databases were analyzed with ArrayTrack v3.3.0 (National Center for Toxicological Research, Jefferson, AR, USA).

For the GSEA, a selection of 144 gene sets from gene ontology (GO) databases was based on biological relevance (Table I). Gene sets were considered to be significantly differently regulated with a false discovery rate (FDR) when $\mathrm{q}<0.05$.

\section{Results}

Single gene analysis revealed that 119 genes were downregulated and 55 genes were upregulated by $>1.5$-fold change (unadjusted p-value $<0.01$ ) across all the treatments (Fig. 1 and an exhaustive list of the differentially expressed genes can be found in Table II). KEGG and PathArt databases indicated that the 54 genes that were downregulated only by RPM treatment were mostly involved in cell cycle regulation (p53- and p21-mediated pathways), in cytoskeleton modeling, cell junctions and cell signaling via integrins, IL- 1 , and TGF- $\beta$. Within the list of individual genes that were downregulated after IR or RPM and IR treatments, no clear pathway was found. On the other hand, in the 52 genes that were upregulated following RPM and RPM and IR treatments, interleukin signaling (IL-11 and MMP) and glutathione metabolism were the most prominent pathways affected. Some genes were differentially expressed by RPM and RPM and IR, however, only a few genes were common between IR and RPM and IR. Six genes were upregulated (S1p3, Rab11b, Ptger3, Vldlr, Cnn1 and Serping1) and only one predicted gene of unknown function was downregulated (Gm13668) in both irradiated treatments (IR and RPM and IR). The upregulated genes were mostly membrane proteins, G-protein coupled (S1p3 and Ptger3) or involved in ligand endocytosis (Rab1lb and Vldlr). Cnn1 and Serping1, involved in cytoskeleton organization and peptidase inhibition, respectively, were both upregulated in all the treatments, including RPM. 
Table I. List of the 144 gene sets selected for GSEA.

Gene set description

Gene Ontology

Actin binding

Actin cytoskeleton

GO:0003779

Activation of JNK activity

Activation of MAPK activity

Adherens junction

Anti-apoptosis

Antioxidant activity

Apoptosis GO

Base excision repair

Calcium ion binding

Calcium ion transport

Caspase activation

Cell-cell adhesion

Cell-cell signaling

Cell cycle arrest

Cell cycle

Cell cycle process

Cell junction

Cell matrix adhesion

Cellular respiration

Centrosome

Chaperone binding

Chromatin

Chromosome

Collagen

Cortical cytoskeleton

Cytokine activity

Cytoskeletal protein binding

Cytoskeleton

DNA damage checkpoint

DNA integrity checkpoint

DNA repair

Double-strand break repair

Electron transport

Embryonic development

Endoplasmic reticulum

Excretion

Extracellular matrix

Focal adhesion

G-protein coupled receptor activity

G-protein coupled receptor

protein signaling pathway

G-protein signaling coupled to IP3 second

messenger phospholipase $\mathrm{C}$ activating

G1 phase

G1/S transition of mitotic cell cycle

G2/M transition of mitotic cell cycle

Glutathione transferase activity

Golgi apparatus

GTPase regulator activity

Histone modification

Hormone activity

GO:0007257

GO:0000187

GO:0005912

GO:0006916

GO:0016209

GO:0006915

GO:0006284

GO:0005509

GO:0006816

GO:0006919

GO:0016337

GO:0007267

GO:0007050

GO:0007049

GO:0022402

GO:0030054

GO:0007160

GO:0045333

GO:0051087

GO:0000785

GO:0005694

GO:0005581

GO:0030863

GO:0005125

GO:0008092

GO:0005856

GO:0000077

GO:0031570

GO:0006281

GO:0006302

GO:0006118

GO:0009790

GO:0005783

GO:0007588

GO:0031012

GO:0005925

GO:0004930

GO:0007186

GO:0007200

GO:0051318

GO:0000082

GO:0000086

GO:0004364

GO:0005794

GO:0030695

GO:0016570

GO:0005179
Table I. Continued.

Gene set description

Gene Ontology

Inositol or phosphatidylinositol

GO:0004428

kinase activity

Inositol or phosphatidylinositol

GO:0004437

GO:0005813 phosphatase activity

Inositol or phosphatidylinositol

phosphodiesterase activity

Insulin receptor signaling pathway

Integrin binding

Intercellular junction

Ion channel activity

JAK/STAT cascade

JNK cascade

Lamellipodium

Lipid binding

M phase

Magnesium ion binding

MAP kinase activity

MAPKKK cascade

Microtubule

Microtubule cytoskeleton

Mitochondrial inner membrane

Mitochondrial respiratory chain

Mitochondrion

Motor activity

Negative regulation of apoptosis

Negative regulation of cell adhesion

Negative regulation of cell cycle

Negative regulation of cell proliferation

Negative regulation of

cellular metabolic process

Negative regulation of signal transduction

Negative regulation of transcription

Negative regulation of translation

Nuclear pore

Nucleolus

Nucleus

Oligosaccharide metabolic process

Phosphoinositide-mediated signaling

Phospholipase activity

Phospholipid binding

Phosphorylation

Positive regulation of caspase activity

Positive regulation of cell adhesion

Positive regulation of cell cycle

Positive regulation of cell proliferation

Positive regulation of JNK activity

Positive regulation of MAP kinase activity

Positive regulation of

protein metabolic process

Positive regulation of signal transduction

Positive regulation of transcription

Positive regulation of translation
GO:0004434

GO:0008286

GO:0005178

GO:0005911

GO:0005216

GO:0007259

GO:0007254

GO:0030027

GO:0008289

GO:0000279

GO:0000287

GO:0004707

GO:0000165

GO:0005874

GO:0015630

GO:0005743

GO:0005746

GO:0005739

GO:0003774

GO:0043066

GO:0007162

GO:0045786

GO:0008285

GO:0031324

GO:0009968

GO:0016481

GO:0017148

GO:0005643

GO:0005730

GO:0005634

GO:0009311

GO:0048015

GO:0004620

GO:0005543

GO:0016310

GO:0043280

GO:0045785

GO:0045787

GO:0008284

GO:0043507

GO:0043406

GO:0051247

GO:0009967

GO:0045941

GO:0045727 
Table I. Continued.

\begin{tabular}{|c|c|}
\hline Gene set description & Gene Ontology \\
\hline Post-translational protein modification & GO:0043687 \\
\hline Potassium ion transport & GO:0006813 \\
\hline Programmed cell death & GO:0012501 \\
\hline Protein folding & GO:0006457 \\
\hline Protein kinase activity & GO:0004672 \\
\hline Protein kinase cascade & GO:0007243 \\
\hline Protein metabolic process & GO:0019538 \\
\hline Protein modification process & GO:0006464 \\
\hline Protein/RNA complex assembly & GO:0022618 \\
\hline Protein serine/threonine kinase activity & GO:0004674 \\
\hline Protein ubiquitination & GO:0016567 \\
\hline Proteolysis & GO:0006508 \\
\hline RAS GTPase activator activity & GO:0005099 \\
\hline RAS GTPase binding & GO:0017016 \\
\hline Receptor binding & GO:0005102 \\
\hline Regulation of apoptosis & GO:0042981 \\
\hline Replication fork & GO:0005657 \\
\hline Respiratory chain complex I & GO:0045271 \\
\hline Response to DNA damage stimulus & GO:0006974 \\
\hline Response to ionizing radiation & GO:0010212 \\
\hline Response to radiation & GO:0009314 \\
\hline Response to stress & GO:0006950 \\
\hline RHO GTPase activator activity & GO:0005100 \\
\hline RHO protein signal transduction & GO:0007266 \\
\hline Rhodopsin-like receptor activity & GO:0001584 \\
\hline RNA helicase activity & GO:0003724 \\
\hline RNA processing & GO:0006396 \\
\hline RNA splicing & GO:0008380 \\
\hline Ruffle & GO:0001726 \\
\hline S phase & GO:0051320 \\
\hline Second messenger-mediated signaling & GO:0019932 \\
\hline Small conjugated protein ligase activity & GO:0019787 \\
\hline Small GTPase-mediated signal transduction & GO:0007264 \\
\hline Sodium channel activity & GO:0005272 \\
\hline Spindle & GO:0005819 \\
\hline Spliceosome & GO:0005681 \\
\hline Structural constituent of cytoskeleton & GO:0005200 \\
\hline Structural constituent of ribosome & GO:0003735 \\
\hline Tight junction & GO:0005923 \\
\hline Transcription & GO:0006350 \\
\hline Translation & GO:0006412 \\
\hline Transmembrane receptor protein & GO:0019199 \\
\hline \multicolumn{2}{|l|}{ kinase activity } \\
\hline Transmembrane transporter activity & GO:0022857 \\
\hline T-RNA metabolic process & GO:0006399 \\
\hline Ubiquitin cycle & GO:0006512 \\
\hline Ubiquitin protein ligase activity & GO:0004842 \\
\hline Voltage-gated channel activity & GO:0022832 \\
\hline
\end{tabular}

GSEA, gene set enrichment analysis.

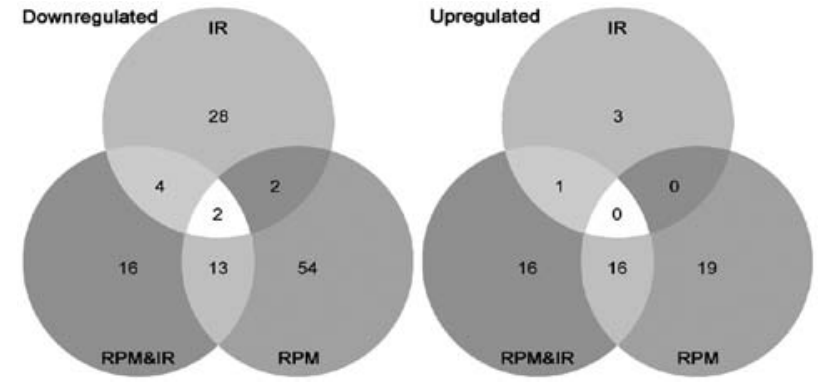

Figure 1. Venn diagram showing the number of downregulated (left) or upregulated (right) genes in murine fetal fibroblasts following one of the three space simulation treatments $(\mathrm{p}<0.01$, fold change $>1.5)$ : chronic exposure to low dose of ionizing radiation (IR), simulated microgravity (RPM) or a combination of RPM and IR.

In contrast to the results obtained by SGA, GSEA revealed a high impact of IR on coordinately differentially expressed genes. A total of 63 gene sets were significantly downregulated following chronic low-dose irradiation. Of the 63 genes, 30 were exclusively enriched in irradiated samples (Fig. 2), although this number may be an overestimation due to redundancy between some of the gene sets. The gene sets that were specifically downregulated after irradiation conditions are mostly involved in DNA damage response, cell signaling, cell cycle, RNA processing and protein turnover (Table III). Moreover, we detected significantly downregulated gene sets involved in cell signaling, cell cycle, transcription, protein turnover, cell shape, adhesion, motility and communication for all the treatments. Of note, two gene sets involved in oxidative phosphorylation were significantly downregulated solely in the RPM and IR samples. No gene set was significantly upregulated in any of the treatments.

\section{Discussion}

In this study, primary cultures of murine fetal fibroblasts were chronically exposed $(65 \mathrm{~h})$ to simulated space conditions including simulated microgravity via RPM and a low-dose mixture of neutrons and gamma-rays (IR). The duration of the experiment was chosen to allow cellular adaptation to the simulated microgravity environment for instance for cytoskeleton remodeling $(13,14)$, in order to decrease the primary stress response mechanisms and to better characterize the effects of chronic exposure to these conditions. Microarrays were performed on RNA harvested from CTRL, IR, RPM and RPM and IR conditions. Microarrays generate a substantial amount of information on the gene expression pattern of cells subjected to a defined treatment. However, a $<2$-fold difference in the gene expression is often not sufficient to meet the requirements for statistical significance (8). Identification of moderate gene expression differences in groups of genes acting together in a cell process can nevertheless be achieved by means of GSEA. For this reason, we analyzed our microarray output data using the single gene analysis method as well as GSEA.

The RPM has a dominant impact on single gene expression. The SGA method revealed a significant impact of $65 \mathrm{~h}$ of simulated microgravity on gene expression in murine fetal fibroblasts. The combination of RPM and IR triggered a 
Table II. Down- and upregulated genes following IR, RPM or RPM and IR treatments $(\mathrm{p}<0.001$, fold change $>1.5)$.

A, Down- and upregulated genes following IR

\begin{tabular}{|c|c|c|c|}
\hline Gene symbol & GenBank & p-value & FC \\
\hline Rab11b & NM_008997 & $5,48 \mathrm{E}-03$ & $-2,026$ \\
\hline Csgalnact1 & NM_172753 & $9,04 \mathrm{E}-03$ & $-1,989$ \\
\hline Smarca5 & NM_053124 & $4,08 \mathrm{E}-03$ & $-1,986$ \\
\hline Tceb3 & NM_013736 & $6,85 \mathrm{E}-03$ & $-1,953$ \\
\hline Serping1 & NM_009776 & $1,39 \mathrm{E}-03$ & $-1,948$ \\
\hline Ppp1r2 & NM_025800 & $9,20 \mathrm{E}-03$ & $-1,801$ \\
\hline Ptgfrn & NM_011197 & $4,38 \mathrm{E}-03$ & $-1,801$ \\
\hline Dnaja1 & NM_008298 & $6,13 \mathrm{E}-03$ & $-1,772$ \\
\hline Arhgap24 & NM_029270 & $6,13 \mathrm{E}-03$ & $-1,767$ \\
\hline Thra & NM_178060 & $3,67 \mathrm{E}-04$ & $-1,728$ \\
\hline Itga8 & NM_001001309 & $9,74 \mathrm{E}-03$ & $-1,718$ \\
\hline Gpr108 & NM_030084 & $2,40 \mathrm{E}-03$ & $-1,696$ \\
\hline Zfp346 & NM_012017 & $1,54 \mathrm{E}-04$ & $-1,672$ \\
\hline Rbmx & NM_011252 & $1,04 \mathrm{E}-03$ & $-1,655$ \\
\hline B4galt6 & NM_019737 & $9,25 \mathrm{E}-03$ & $-1,638$ \\
\hline ВC003331 & NM_145511 & $5,04 \mathrm{E}-03$ & $-1,637$ \\
\hline Vldlr & NM_013703 & $3,68 \mathrm{E}-03$ & $-1,636$ \\
\hline Unc93b1 & NM_019449 & $5,57 \mathrm{E}-04$ & $-1,625$ \\
\hline Pip4k2a & NM_008845 & 3,79E-04 & $-1,622$ \\
\hline Mgll & NM_001166251 & $2,52 \mathrm{E}-03$ & $-1,620$ \\
\hline ВC005624 & NM_144885 & $2,10 \mathrm{E}-03$ & $-1,619$ \\
\hline S1pr3 & NM_010101 & $2,64 \mathrm{E}-03$ & $-1,613$ \\
\hline Prkcd & NM_011103 & $3,32 \mathrm{E}-03$ & $-1,583$ \\
\hline Cnn1 & NM_009922 & $4,26 \mathrm{E}-03$ & $-1,575$ \\
\hline P2ry2 & NM_008773 & $6,80 \mathrm{E}-03$ & $-1,566$ \\
\hline Saps1 & NM_172894 & $8,65 \mathrm{E}-03$ & $-1,566$ \\
\hline Casc4 & NM_177054 & $4,45 \mathrm{E}-03$ & $-1,559$ \\
\hline Opa1 & NM_133752 & $7,47 \mathrm{E}-03$ & $-1,552$ \\
\hline Emb & NM_010330 & $5,84 \mathrm{E}-04$ & $-1,551$ \\
\hline Cyb5d1 & NM_001045525 & $5,23 \mathrm{E}-03$ & $-1,549$ \\
\hline Ptger3 & NM_011196 & $1,41 \mathrm{E}-03$ & $-1,549$ \\
\hline Usp30 & NM_001033202 & $1,36 \mathrm{E}-03$ & $-1,543$ \\
\hline Tbc1d $2 b$ & NM_194334 & $6,00 \mathrm{E}-03$ & $-1,539$ \\
\hline Cyld & NM_001128169 & $2,57 \mathrm{E}-03$ & $-1,530$ \\
\hline Trip4 & NM_019797 & $8,21 \mathrm{E}-03$ & $-1,520$ \\
\hline Luzp1 & NM_024452 & $9,75 \mathrm{E}-03$ & $-1,502$ \\
\hline Gm13668 & XR_032757 & $6,87 \mathrm{E}-04$ & 1,856 \\
\hline Hist1h2ao & NM_001177544 & $3,69 \mathrm{E}-03$ & 1,710 \\
\hline Bmyc & NM_023326 & $3,25 \mathrm{E}-03$ & 1,575 \\
\hline 4930458L03Rik & NM_030047 & $1,32 \mathrm{E}-03$ & 1,523 \\
\hline
\end{tabular}

B, Down- and upregulated genes following RPM

\begin{tabular}{llll}
\hline Dmpk & NM_032418 & $2,73 \mathrm{E}-05$ & $-2,522$ \\
Myh10 & NM_175260 & $1,51 \mathrm{E}-03$ & $-2,485$ \\
Myh9 & NM_022410 & $2,08 \mathrm{E}-03$ & $-2,432$ \\
Maob & NM_172778 & $1,48 \mathrm{E}-04$ & $-2,335$ \\
Slc38a4 & NM_027052 & $9,81 \mathrm{E}-04$ & $-2,270$ \\
Cnn1 & NM_009922 & $4,53 \mathrm{E}-05$ & $-2,147$ \\
Adh1 & NM_007409 & 2,01E-04 & $-2,126$ \\
Serping1 & NM_009776 & $5,85 \mathrm{E}-04$ & $-2,095$
\end{tabular}

Table II. Continued.

\begin{tabular}{|c|c|c|c|}
\hline Gene symbol & GenBank & p-value & FC \\
\hline $\operatorname{Actg} 2$ & NM_009610 & $3,08 \mathrm{E}-05$ & $-2,089$ \\
\hline Ccnb2 & NM_007630 & $2,94 \mathrm{E}-04$ & $-2,071$ \\
\hline Kif20a & NM_001166406 & $1,35 \mathrm{E}-04$ & $-2,023$ \\
\hline Gjb2 & NM_008125 & $1,16 \mathrm{E}-04$ & $-2,014$ \\
\hline Anln & NM_028390 & $8,66 \mathrm{E}-04$ & $-2,001$ \\
\hline Nfix & NM_001081981 & $3,95 \mathrm{E}-03$ & $-1,964$ \\
\hline Itga8 & NM_001001309 & 2,19E-03 & $-1,963$ \\
\hline Pygb & NM_153781 & $1,46 \mathrm{E}-03$ & $-1,913$ \\
\hline Bub1 & NM_001113179 & $3,71 \mathrm{E}-05$ & $-1,881$ \\
\hline Ly6c1 & NM_010741 & 9,89E-04 & $-1,879$ \\
\hline ND4L & ENSMUST00000084013 & $2,03 \mathrm{E}-05$ & $-1,843$ \\
\hline Myl9 & NM_172118 & $1,95 \mathrm{E}-04$ & $-1,830$ \\
\hline Actn4 & NM_021895 & $8,48 \mathrm{E}-03$ & $-1,819$ \\
\hline Itgbl1 & NM_145467 & 8,49E-03 & $-1,814$ \\
\hline Efemp1 & NM_146015 & $6,17 \mathrm{E}-04$ & $-1,801$ \\
\hline D17H6S56E-5 & L78788 & $2,29 \mathrm{E}-07$ & $-1,791$ \\
\hline Plk1 & NM_011121 & $1,55 \mathrm{E}-03$ & $-1,774$ \\
\hline ND4L & ENSMUST00000084013 & $3,76 \mathrm{E}-05$ & $-1,750$ \\
\hline Susd2 & NM_027890 & $2,62 \mathrm{E}-04$ & $-1,736$ \\
\hline Ly6c 2 & NM_001099217 & $7,26 \mathrm{E}-04$ & $-1,731$ \\
\hline Ucp2 & NM_011671 & 4,37E-04 & $-1,717$ \\
\hline Cenpa & NM_007681 & $3,25 \mathrm{E}-03$ & $-1,713$ \\
\hline Nuf2 & NM_023284 & 6,69E-04 & $-1,711$ \\
\hline Rbmx & NM_011252 & 7,19E-04 & $-1,692$ \\
\hline Kif2c & NM_134471 & $2,28 \mathrm{E}-03$ & $-1,687$ \\
\hline Rpl2211 & NM_026517 & $9,88 \mathrm{E}-03$ & $-1,678$ \\
\hline Ly6a & NM_010738 & 8,47E-03 & $-1,671$ \\
\hline Pkp2 & NM_026163 & $1,21 \mathrm{E}-04$ & $-1,667$ \\
\hline Tgfb1i1 & NM_009365 & $6,54 \mathrm{E}-03$ & $-1,652$ \\
\hline Acta1 & NM_009606 & 7,19E-06 & $-1,644$ \\
\hline Gas 213 & NM_001033331 & $5,26 \mathrm{E}-04$ & $-1,643$ \\
\hline Lrrc17 & NM_028977 & 4,37E-03 & $-1,642$ \\
\hline 2810417H13Rik & NM_026515 & $3,58 \mathrm{E}-03$ & $-1,640$ \\
\hline Lpar4 & NM_175271 & $3,20 \mathrm{E}-03$ & $-1,639$ \\
\hline Dlgap5 & NM_144553 & $1,76 \mathrm{E}-03$ & $-1,622$ \\
\hline Hgf & NM_010427 & 1,71E-03 & $-1,611$ \\
\hline Trp53inp2 & NM_178111 & $1,30 \mathrm{E}-03$ & $-1,605$ \\
\hline Cyb5r3 & NM_029787 & $1,06 \mathrm{E}-03$ & $-1,603$ \\
\hline Mfap2 & NM_008546 & $6,77 \mathrm{E}-04$ & $-1,600$ \\
\hline Cyp1b1 & NM_009994 & $5,70 \mathrm{E}-03$ & $-1,597$ \\
\hline Trpv2 & NM_011706 & $4,75 \mathrm{E}-03$ & $-1,596$ \\
\hline Kif23 & NM_024245 & $1,56 \mathrm{E}-03$ & $-1,591$ \\
\hline Sh3pxd2a & NM_008018 & 1,37E-03 & $-1,566$ \\
\hline ND2 & ENSMUST00000082396 & $1,35 \mathrm{E}-03$ & $-1,564$ \\
\hline Tgfb3 & NM_009368 & $1,15 \mathrm{E}-03$ & $-1,562$ \\
\hline Scd2 & NM_009128 & $5,24 \mathrm{E}-03$ & $-1,554$ \\
\hline Dner & NM_152915 & $1,80 \mathrm{E}-03$ & $-1,546$ \\
\hline Pdgfrl & NM_026840 & 4,88E-04 & $-1,543$ \\
\hline Cenpm & NM_025639 & $6,47 \mathrm{E}-03$ & $-1,539$ \\
\hline Ppp1r3c & NM_016854 & $1,04 \mathrm{E}-03$ & $-1,536$ \\
\hline Fam114a1 & NM_026667 & $1,68 \mathrm{E}-03$ & $-1,533$ \\
\hline D2Ertd750e & NM_026412 & $7,48 \mathrm{E}-04$ & $-1,533$ \\
\hline $\mathrm{Nkd} 2$ & NM_028186 & $7,87 \mathrm{E}-03$ & $-1,531$ \\
\hline
\end{tabular}


Table II. Continued.

\begin{tabular}{|c|c|c|c|c|c|c|c|}
\hline \multirow{2}{*}{$\begin{array}{l}\text { Gene symbol } \\
\text { Nov }\end{array}$} & \multirow{2}{*}{$\begin{array}{l}\text { GenBank } \\
\text { NM_010930 }\end{array}$} & \multirow{2}{*}{$\frac{\mathrm{p} \text {-value }}{9,41 \mathrm{E}-03}$} & \multirow{2}{*}{$\frac{\mathrm{FC}}{-1,529}$} & \multicolumn{4}{|c|}{ C, Down- and upregulated genes following RPM and IR } \\
\hline & & & & Gene symbol & GenBank & p-value & $\mathrm{FC}$ \\
\hline Tgm2 & NM_009373 & $2,62 \mathrm{E}-03$ & $-1,525$ & & & & \\
\hline Nucb2 & NM_001130479 & $7,36 \mathrm{E}-03$ & $-1,518$ & Cnn1 & NM_009922 & $6,32 \mathrm{E}-06$ & $-2,494$ \\
\hline 5730469M10Rik & $\mathrm{BC} 056635$ & $1,03 \mathrm{E}-03$ & $-1,516$ & Serping1 & NM_009776 & $1,65 \mathrm{E}-04$ & $-2,337$ \\
\hline Ccna2 & NM_009828 & $8,65 \mathrm{E}-03$ & $-1,514$ & Dmpk & NM_032418 & $1,36 \mathrm{E}-04$ & $-2,206$ \\
\hline Maged2 & NM_030700 & $6,56 \mathrm{E}-03$ & $-1,512$ & $\operatorname{Actg} 2$ & NM_009610 & $1,44 \mathrm{E}-05$ & $-2,203$ \\
\hline Eif4b & NM_145625 & 7,83E-03 & $-1,512$ & Adh1 & NM_007409 & $2,24 \mathrm{E}-04$ & $-2,107$ \\
\hline Sepx 1 & NM_013759 & $2,65 \mathrm{E}-04$ & $-1,506$ & Rab11b & NM_008997 & $4,88 \mathrm{E}-03$ & $-2,051$ \\
\hline Shisa4 & NM_175259 & $5,60 \mathrm{E}-03$ & $-1,503$ & Itgbl1 & NM_145467 & $2,48 \mathrm{E}-03$ & $-2,041$ \\
\hline St3gal5 & NM_011375 & $8,29 \mathrm{E}-03$ & $-1,502$ & Gjb2 & NM_008125 & $1,20 \mathrm{E}-04$ & $-2,009$ \\
\hline Fhl5 & NM_021318 & $232 \mathrm{E}-04$ & $-1,002$ & Srpx & NM_016911 & $1,74 \mathrm{E}-05$ & $-1,882$ \\
\hline & NIM_021318 & $2,02 \mathrm{E}-\mathrm{U} 4$ & $-1,002$ & My19 & NM_172118 & $1,72 \mathrm{E}-04$ & $-1,844$ \\
\hline Serpinb9e & NM_011456 & $2,02 \mathrm{E}-03$ & 2,514 & S1pr3 & NM_010101 & $4,04 \mathrm{E}-04$ & $-1,824$ \\
\hline Gst $\alpha 1$ & NM_008181 & $1,59 \mathrm{E}-05$ & 2,232 & Maob & NM_172778 & $2,99 \mathrm{E}-03$ & $-1,814$ \\
\hline Taf1d & ВC056964 & 1,32E-03 & 2,223 & Tmem45a & NM_019631 & $3,16 \mathrm{E}-04$ & $-1,793$ \\
\hline Gst $\alpha 1$ & NM_008181 & $2,15 \mathrm{E}-05$ & 2,210 & Pdgfrl & NM_026840 & $3,28 \mathrm{E}-05$ & $-1,7722$ \\
\hline $\operatorname{Pr} 12 \mathrm{c} 3$ & NM_011118 & $2,70 \mathrm{E}-05$ & 2,209 & Nov & NM_010930 & $1,36 \mathrm{E}-03$ & $-1,749$ \\
\hline Snhg1 & AK051045 & $5,45 \mathrm{E}-06$ & 2,192 & Pigc & NM_026078 & $5,96 \mathrm{E}-03$ & $-1,691$ \\
\hline $\operatorname{Pr} 12 c 5$ & NM_181852 & 2,79E-04 & 2,179 & Il1r1 & NM_008362 & $5,64 \mathrm{E}-03$ & $-1,690$ \\
\hline Malat1 & NR_002847 & $1,90 \mathrm{E}-04$ & 2,139 & Vldlr & NM_013703 & 2,37E-03 & $-1,687$ \\
\hline Il1rl1 & NM_001025602 & $2,81 \mathrm{E}-04$ & 2,061 & Susd2 & NM_027890 & $6,17 \mathrm{E}-04$ & $-1,652$ \\
\hline Snhg1 & AK051045 & $1,78 \mathrm{E}-05$ & 1,967 & Ptger3 & NM_011196 & 4,43E-04 & $-1,651$ \\
\hline Gm10639 & NM_001122660 & $2,00 \mathrm{E}-04$ & 1,908 & Lysmd3 & NM_030257 & 2,93E-03 & $-1,650$ \\
\hline Sema7a & NM_011352 & $5,57 \mathrm{E}-04$ & 1,870 & Fhl1 & NM_001077361 & $1,90 \mathrm{E}-08$ & $-1,629$ \\
\hline Lce1h & NM_026335 & $6,44 \mathrm{E}-03$ & 1,841 & Cyp1b1 & NM_009994 & $4,48 \mathrm{E}-03$ & $-1,625$ \\
\hline Taf1d & ВC056964 & $7,18 \mathrm{E}-04$ & 1,812 & Plk1 & NM_011121 & $6,41 \mathrm{E}-03$ & $-1,600$ \\
\hline Crct1 & NM_028798 & $3,49 \mathrm{E}-04$ & 1,802 & St3gal5 & NM_011375 & $3,71 \mathrm{E}-03$ & $-1,583$ \\
\hline Gm8074 & XM_983501 & $3,90 \mathrm{E}-04$ & 1,799 & Rab13 & NM_026677 & 7,09E-04 & $-1,581$ \\
\hline Lsm1 & NM_026032 & $1,31 \mathrm{E}-03$ & 1,794 & Snta1 & NM_009228 & 4,11E-05 & $-1,577$ \\
\hline 2310002L13Rik & ENSMUST00000025390 & $4,85 \mathrm{E}-04$ & 1,771 & Aqp1 & NM_007472 & $5,14 \mathrm{E}-03$ & $-1,556$ \\
\hline Sirt7 & NM_153056 & $4,15 \mathrm{E}-04$ & 1,760 & Cpa6 & NM_177834 & $4,01 \mathrm{E}-03$ & $-1,554$ \\
\hline Serpinb9b & NM_011452 & 4,07E-06 & 1,734 & Nosip & NM_025533 & $1,35 \mathrm{E}-03$ & $-1,540$ \\
\hline Snord $14 \mathrm{e}$ & NR_028275 & $7,22 \mathrm{E}-04$ & 1,704 & Pla2g 16 & NM_139269 & $5,13 \mathrm{E}-03$ & $-1,532$ \\
\hline Gst $\alpha 2$ & NM_008182 & $7,50 \mathrm{E}-07$ & 1,700 & Lmod1 & NM_053106 & $3,01 \mathrm{E}-03$ & $-1,523$ \\
\hline Ppbp & NM_023785 & $5,04 \mathrm{E}-03$ & 1,691 & Zcchc17 & NM_153160 & 4,13E-03 & $-1,519$ \\
\hline Hsd3b6 & NM_013821 & $1,21 \mathrm{E}-04$ & 1,684 & Islr & NM_012043 & $1,27 \mathrm{E}-03$ & $-1,509$ \\
\hline Snord14d & NR_028274 & 7,27E-04 & 1,679 & 6330406I15Rik & BC116246 & $1,22 \mathrm{E}-04$ & $-1,502$ \\
\hline Hmox1 & NM_010442 & $5,24 \mathrm{E}-06$ & 1,678 & Serpinb9e & NM_011456 & 7,91E-04 & 2,818 \\
\hline Clcf1 & NM_019952 & $1,97 \mathrm{E}-04$ & 1,671 & Slc40a1 & NM_016917 & $1,83 \mathrm{E}-05$ & 2,670 \\
\hline Snord14d & NR_028274 & 7,39E-04 & 1,667 & Taf1d & ВС056964 & $2,85 \mathrm{E}-04$ & 2,595 \\
\hline Procr & NM_011171 & $2,00 \mathrm{E}-03$ & 1,649 & Snhg1 & AK051045 & $8,57 \mathrm{E}-06$ & 2,126 \\
\hline Hist1h4i & NM_175656 & 4,77E-03 & 1,635 & $\operatorname{Prl} 2 \mathrm{c} 3$ & NM_011118 & $8,34 \mathrm{E}-05$ & 2,037 \\
\hline Dusp4 & NM_176933 & $4,07 \mathrm{E}-03$ & 1,626 & Gst $\alpha 1$ & NM_008181 & $1,21 \mathrm{E}-04$ & 1,938 \\
\hline Mmp10 & NM_019471 & $6,51 \mathrm{E}-04$ & 1,587 & Procr & NM_011171 & $1,83 \mathrm{E}-04$ & 1,935 \\
\hline Cops3 & NM_011991 & $1,24 \mathrm{E}-03$ & 1,584 & Gst $\alpha 1$ & NM_008181 & $1,60 \mathrm{E}-04$ & 1,921 \\
\hline Gas5 & NR_002840 & $3,87 \mathrm{E}-03$ & 1,573 & Mmp13 & NM_008607 & $5,88 \mathrm{E}-04$ & 1,894 \\
\hline Chrna1 & NM_007389 & $1,19 \mathrm{E}-03$ & 1,565 & Malat1 & NR_002847 & $1,05 \mathrm{E}-03$ & 1,873 \\
\hline Ifrd 1 & NM_013562 & $1,44 \mathrm{E}-03$ & 1,556 & Serpinb9g & NM_011455 & $5,62 \mathrm{E}-03$ & 1,865 \\
\hline D4Wsu53e & ВC043057 & $2,60 \mathrm{E}-03$ & 1,515 & Snhg1 & AK051045 & $5,03 \mathrm{E}-05$ & 1,848 \\
\hline S100a7a & NM_199422 & $8,26 \mathrm{E}-03$ & 1,513 & Serpinb9g & NM_011455 & $5,24 \mathrm{E}-03$ & 1,801 \\
\hline Scarna17 & NR_028560 & $6,25 \mathrm{E}-04$ & 1,512 & 310002L13Rik & ENSMUST00000025390 & $4,28 \mathrm{E}-04$ & 1,785 \\
\hline Scarna17 & NR_028560 & $6,25 \mathrm{E}-04$ & 1,512 & Gm8074 & XM_983501 & $6,00 \mathrm{E}-04$ & 1,750 \\
\hline
\end{tabular}

Table II. Continued. 
Table II. Continued.

\begin{tabular}{|c|c|c|c|}
\hline Gene symbol & GenBank & p-value & $\mathrm{FC}$ \\
\hline Peg10 & NM_130877 & $1,83 \mathrm{E}-03$ & 1,746 \\
\hline Mamdc2 & NM_174857 & 7,44E-04 & 1,744 \\
\hline Il11 & NM_008350 & $9,82 \mathrm{E}-03$ & 1,728 \\
\hline Mmp3 & NM_010809 & $8,35 \mathrm{E}-03$ & 1,724 \\
\hline Fabp7 & NM_021272 & 2,79E-03 & 1,693 \\
\hline Serpinb9b & NM_011452 & 7,99E-06 & 1,681 \\
\hline Taf1d & ВС056964 & $2,40 \mathrm{E}-03$ & 1,667 \\
\hline Gmnn & AF068780 & $7,71 \mathrm{E}-03$ & 1,620 \\
\hline Gm10639 & NM_001122660 & $2,64 \mathrm{E}-03$ & 1,610 \\
\hline Dusp4 & NM_176933 & $5,31 \mathrm{E}-03$ & 1,596 \\
\hline $\operatorname{Bcl} 2111$ & NM_207680 & $9,00 \mathrm{E}-03$ & 1,595 \\
\hline Ctu1 & NM_145582 & $1,01 \mathrm{E}-03$ & 1,594 \\
\hline Gsta2 & NM_008182 & $3,74 \mathrm{E}-06$ & 1,591 \\
\hline Hsd3b6 & NM_013821 & $5,25 \mathrm{E}-04$ & 1,561 \\
\hline Gm13668 & XR_032757 & $8,44 \mathrm{E}-03$ & 1,553 \\
\hline Ang2 & NM_007449 & $1,54 \mathrm{E}-03$ & 1,548 \\
\hline Scarna17 & NR_028560 & $3,97 \mathrm{E}-04$ & 1,545 \\
\hline Scarna17 & NR_028560 & $3,97 \mathrm{E}-04$ & 1,545 \\
\hline Hmox1 & NM_010442 & $3,84 \mathrm{E}-05$ & 1,541 \\
\hline Serpinb9f & NM_183197 & $9,11 \mathrm{E}-04$ & 1,540 \\
\hline Opa3 & NM_207525 & $1,23 \mathrm{E}-03$ & 1,540 \\
\hline Ormdl3 & NM_025661 & $8,94 \mathrm{E}-03$ & 1,505 \\
\hline
\end{tabular}

IR, ionizing radiation; RPM, random positioning machine.

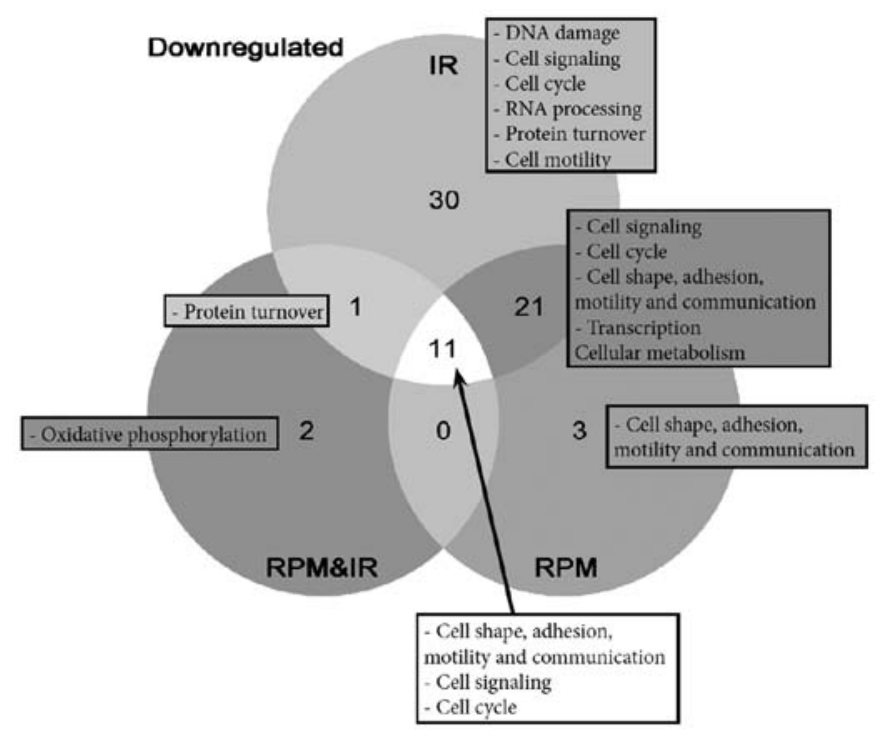

Figure 2. Venn diagram showing the number of gene sets significantly downregulated in murine fetal fibroblasts following one of the three space simulation treatments: chronic exposure to a low dose of irradiation (IR), simulated microgravity (RPM) or a combination of RPM and IR. Boxes include the cellular pathways in which these gene sets are involved.

differential expression of fewer genes than RPM alone. Only a few genes had an altered expression in IR samples, suggesting that such a low dose of radiation exerted a moderate impact on the expression of individual genes. It was also noted that only a few genes were commonly differentially expressed in all irradiated treatments (IR and RPM and IR), of which there were only six known genes, all upregulated (S1p3, Rab11b, Ptger3, Vldlr, Cnn1 and Serping1), with most of them being involved in cell signaling. No explanation can be provided for the fact that few genes were commonly up- or downregulated in the irradiated treatments (with or without RPM). However the strong effect of RPM may have concealed a more subtle effect of IR, making it statistically less significant.

Among the upregulated genes following RPM treatment, glutathione-S-transferases $\alpha 1$ and 2 (Gst $\alpha 1$ and Gst $\alpha 2)$ were prominent enzymes for the detoxification of breakdown products of oxidative stress (15). However, since the Affymetrix arrays cannot distinguish between the two isoforms due to their very high sequence homology (97\%), we cannot dismiss the possibility that only one of the two isoforms was actually affected by the treatment. The modifier subunit of glutathionecysteine ligase $(\mathrm{Gclm})$ was significantly upregulated as well. The protein encoded by this gene was shown to play an important role in controlling the rate of glutathione synthesis in murine fetal fibroblasts (16). We also report upregulation of the heme oxygenase 1 (Hmoxl), a cytoprotective enzyme against oxidative stress (17). In murine fibroblasts, its upregulation by curcumin was found to block radiation-induced reactive oxygen species (ROS) generation (18). Notably, these three genes are targets of the nuclear factor-erythroid 2 p45-related factor 2 (Nrf2) which induces transcription of cytoprotective genes containing antioxidant response elements (19). The transcription factor $\mathrm{Nrf} 2$ may therefore play a cytoprotective role against a possible oxidative stress induced by the RPM, which is in line with previous observations of increased oxidative stress in simulated microgravity (20-22).

After RPM treatment, two members of the actin filament family, Actg2 and Actal were downregulated. These genes were described in smooth (23) or skeletal muscles (24), respectively. Calponin 1 ( $\mathrm{Cnnl}$ ), a gene coding for a protein involved in the cytoskeleton organization (25), and four and a half LIM domains 1 (Fhll), which functions in adherens junctions signaling to the cytoskeleton (26), were also downregulated. Notably, the four genes were shown to be regulated by the serum response factor (SRF). SRF was shown to be mediated by the Rho signaling pathway (25-27), which may have been triggered by the RPM. Rho signaling is believed to be an important pathway for focal adhesion assembly and cytoskeleton remodeling in response to cellular tension stress (28) and has been suggested to play a role in the microgravity response (21,29-31). Furthermore, Rho GTPase activities were shown to be increased in dermal fibroblasts subjected to simulated microgravity for 30 and $120 \mathrm{~min}$, thereafter decreasing to reach similar values to those of the CTRL at $48 \mathrm{~h}$ of treatment (32). Our hypothesis is that a $65-\mathrm{h}$ exposure to RPM induced downregulation of the Rho signaling pathway, which decreased the activity of the transcription factor SRF, decreasing in turn the expression of genes involved in cytoskeleton organization ( $\mathrm{Cnnl}$ ) and adherens junctions (Fhll).

IR has a dominant effect on gene sets. At the gene set level, GSEA did not detect any upregulation, except for the structural constituents of the ribosome in IR-treated samples. This result is noteworthy as it did not occur with SGA. Since 
Table III. Downregulated gene sets revealed by GSEA, based on the list of gene sets provided by Fig. 2 .

\begin{tabular}{|c|c|c|}
\hline Treatment & Cell process & Gene set (GO) \\
\hline \multirow[t]{6}{*}{ IR } & DNA damage & $\begin{array}{l}\text { DNA damage checkpoint } \\
\text { DNA repair } \\
\text { Histone modification } \\
\text { Response to DNA damage stimulus } \\
\text { Response to radiation } \\
\text { Response to stress }\end{array}$ \\
\hline & Cell signaling & $\begin{array}{l}\text { Negative regulation of signal transduction } \\
\text { Inositol or phosphatidylinositol kinase activity } \\
\text { Ras GTPase binding } \\
\text { Positive regulation of JNK activity } \\
\text { RHO GTPase activator activity } \\
\text { Protein kinase cascade } \\
\text { Magnesium ion binding } \\
\text { Protein serine/threonine kinase activity } \\
\text { Phosphorylation }\end{array}$ \\
\hline & Cell cycle & $\begin{array}{l}\text { Cell cycle arrest (GO 0007050) } \\
\text { Negative regulation of cell cycle }\end{array}$ \\
\hline & RNA processing & $\begin{array}{l}\text { RNA processing } \\
\text { RNA splicing } \\
\text { Spliceosome } \\
\text { Nuclear pore }\end{array}$ \\
\hline & Protein turnover & $\begin{array}{l}\text { tRNA metabolic process } \\
\text { Post translational protein modification } \\
\text { Endoplasmic reticulum } \\
\text { Golgi apparatus } \\
\text { Portein ubiquitination } \\
\text { Ubiquitin cycle } \\
\text { Ubiquitin protein ligase activity } \\
\text { Small conjugating protein ligase activity }\end{array}$ \\
\hline & Cell motility & Lamellipodium \\
\hline \multirow[t]{5}{*}{$\mathrm{IR}+\mathrm{RPM}$} & Cell signaling & $\begin{array}{l}\text { G protein signaling coupled to IP3 } \\
\text { Phosphoinositide-mediated signaling } \\
\text { RAS GTPase activator activity } \\
\text { GTPase regulator activity } \\
\text { Small GTPase-mediated signal transduction } \\
\text { Transmembrane receptor } \\
\text { protein kinase activity } \\
\text { Protein kinase activity }\end{array}$ \\
\hline & Cell cycle & $\begin{array}{l}\text { Cell cycle (GO 0007049) } \\
\text { Centrosome }\end{array}$ \\
\hline & $\begin{array}{l}\text { Cell shape, adhesion, } \\
\text { motility and communication }\end{array}$ & $\begin{array}{l}\text { Microtubule } \\
\text { Cytoskeletal protein binding } \\
\text { Ruffle } \\
\text { Cell junction } \\
\text { Collagen } \\
\text { Extracellular matrix }\end{array}$ \\
\hline & Transcription & $\begin{array}{l}\text { Positive regulation of transcription } \\
\text { Negative regulation of transcription } \\
\text { RNA helicase activity } \\
\text { Chromosome } \\
\text { Nucleolus }\end{array}$ \\
\hline & Cellular metabolism & Negative regulation of cellular metabolic proc \\
\hline
\end{tabular}


Table III. Continued.

\begin{tabular}{lll}
\hline Treatment & Cell process & Gene set (GO) \\
\hline IR + RPMIR + RPM and IR & Protein turnover & Protein/RNA complex assembly \\
IR + RPM + & Cell shape, adhesion, & Cytoskeleton \\
RPM + IR & motility and communication & Actin binding \\
& & Actin cytoskeleton \\
& & Adherens junction \\
& & Cell matrix adhesion \\
& & Motor activity \\
& Cell signaling & Microtubule cytoskeleton \\
CPM & & Insulin receptor signaling pathway \\
& & Cell cycle process \\
RPM + IR & Cell shape, adhesion, & M phase \\
& motility and communication & Spindle \\
& & Structural constituent of cytoskeleton \\
& Oxidative phosphorylation & Integrin binding \\
& & Receptor binding
\end{tabular}

'+' shows the gene sets commonly differentially expressed between the treatments cited; IR, ionizing radiation; RPM, random positioning machine.

SGA and GSEA are purely statistical methods, it is unlikely that this result originates from an experimental issue, which may have affected both methods. We also examined the gene set selection, however, a screening of all the gene sets of GO provided the same result. Since the experimental design involved long-term irradiation, it is possible that a feedback loop occurred and decreased the expression pattern of the gene sets.

We identified a significant downregulation of 63 gene sets in response to low-dose IR, although single gene analysis did not reveal any important effects. Of the 63 gene sets, 30 were specifically enriched in IR-treated samples (Fig. 2). These latter gene sets are involved in DNA damage response, cell signaling, cell cycle, RNA processing, protein turnover or cell motility. Of note, the DNA damage response gene sets were downregulated, which may be explained by the long duration of continuous irradiation at an extremely slow-dose rate. It is possible that an adaptation mechanism of the cells to irradiation triggered a feedback loop to decrease the expression of these pathways, as was observed at the gene level (SGA) for SRF responsive genes in response to the RPM. Various other gene sets involved in the same cell processes were also enriched in the RPM, and RPM and IR treatments.

Many of the downregulated gene sets are involved in cell signaling, including Rho and Ras GTPases, inositol and phosphatidylinositol, JNK and insulin receptor-mediated pathways. The downregulation of these signaling pathways may lead to an alteration of the cell cycle (33). In addition to its major role in the cell response to radiation $(34,35)$, the regulation of the cell cycle has been shown to be affected by simulated microgravity (36). GSEA revealed that gene sets involved in the positive regulation of the cell cycle were downregulated in all treatments. However, cells that were only irradiated exhibited a significant downregulation of gene sets involved in cell cycle arrest, indicating no trend towards a pro- or anti-proliferative expression profile, while both RPM and RPM and IR showed an anti-proliferative expression profile. We suggest that all the treatments may have induced a general stress response that decreased the expression of cell cycle progression pathways, while irradiation alone also reduced the expression of genes involved in cell cycle arrest. This hypothesis is in agreement with the decreased expression of DNA damage response pathways that we also detected. In RPM and IR, the effect of the RPM may have concealed the cell cycle arrest gene set downregulation.

In addition, many gene sets involved in the composition of the cytoskeleton (actin and microtubule) and inter- (cell junctions) and extracellular connections (extracellular matrix) were affected by all the treatments. While it has been shown in various cell types that cytoskeleton remodeling starts immediately after exposure to simulated or real microgravity (21,29-31), few studies investigated the effects of IR on the cytoskeleton. However, therapeutic doses of irradiation were shown to affect cell permeability of microvascular endothelial cells through Rho-mediated cytoskeleton remodeling (37). More recently, Rho-mediated focal adhesion and fibronectin adhesion were shown to be increased in endothelial cells in response to radiation (38). As Rho GTPases intervene in a number of additional cell pathways (e.g., cell cycle arrest, and regulation of apoptosis) (39), Rho GTPases potentially play a pivotal role in the cell response to simulated space conditions. In agreement with this hypothesis, GSEA revealed that Rho GTPases activity was downregulated in IR-treated samples. Notably, gene sets involved in integrin and receptor binding were specifically downregulated following treatment using the RPM. The results of this study confirm therefore 
that integrins play a significant role in the cellular response to simulated microgravity.

In conclusion, this study has shown that continuous exposure to simulated microgravity affects fetal murine fibroblasts, especially at the single gene level, by increasing the expression of oxidative stress responsive genes and decreasing the expression of genes involved in cytoskeleton remodeling. As far as irradiation is concerned, we detected a decreased expression of gene sets involved in cytoskeleton mechanisms, in cell signaling and DNA damage response after a chronic low-dose rate of irradiation, particularly at the gene set level. The results indicate that the effects of the combination of the two treatments did not result in a synergism between the two separate effects, since many genes or gene sets that were altered by RPM or IR treatment, were not changed by the combined treatment (RPM and IR).

\section{Acknowledgements}

This study was supported by the ESA Topical Team on 'Developmental Biology in Vertebrates' and 4 PRODEX/ESA contracts [C90-303, C90-380, C90-391 and 42-000-90-380].

\section{References}

1. Huijser RH: Desktop RPM: new small size microgravity simulator for the bioscience laboratory. Fokker Space FS-MGR00-017: 1-5, 2000.

2. van Loon JJWA: Some history and use of the random positioning machine, RPM, in gravity related research. Adv Space Res 39: 1161-1165, 2007.

3. Borst A and van Loon J: Technology and developments for the random positioning machine, RPM. Microgravity Sci Technol 21: 287-292, 2009

4. Kraft TF, van Loon JJ and Kiss JZ: Plastid position in arabidopsis columella cells is similar in microgravity and on a randompositioning machine. Planta 211: 415-422, 2000.

5. Villa A, Versari S, Maier JA and Bradamante S: Cell behavior in simulated microgravity: a comparison of results obtained with RWV and RPM. Gravit Space Biol Bull 18: 89-90, 2005.

6. Grimm D, Bauer J, Ulbrich C, et al: Different responsiveness of endothelial cells to vascular endothelial growth factor and basic fibroblast growth factor added to culture media under gravity and simulated microgravity. Tissue Eng Part A 16: 1559-1573, 2010.

7. Mastroleo F, Van Houdt R, Leroy B, et al: Experimental design and environmental parameters affect Rhodospirillum rubrum S1H response to space flight. ISME J 3: 1402-1419, 2009.

8. Shi J and Walker MG: Gene set enrichment analysis (GSEA) for interpreting gene expression profiles. Curr Bioinform 2: 133-137, 2007.

9. Subramanian A, Tamayo P, Mootha VK, et al: Gene set enrichment analysis: A knowledge-based approach for interpreting genome-wide expression profiles. Proc Natl Acad Sci USA 102: 15545-15550, 2005.

10. El-Saghire H, Thierens H,Monsieurs P, Michaux A, Vandevoorde C and Baatout $S$ : Gene set enrichment analysis highlights different gene expression profiles in whole blood samples X-irradiated with low and high doses. Int J Radiat Biol 89: 628-638, 2013.

11. Vanhavere F, Loos M, Plompen AJM, Wattecamps E and Thierens H: A combined use of the BD-PND and BDT bubble detectors in neutron dosimetry. Radiat Meas 29: 573-577, 1998.

12. Cucinotta FA, Kim MH, Willingham V and George KA: Physical and biological organ dosimetry analysis for international space station astronauts. Radiat Res 170: 127-138, 2008.

13. Crawford-Young SJ: Effects of microgravity on cell cytoskeleton and embryogenesis. Int J Dev Biol 50: 183-191, 2006.

14. Meloni MA, Galleri G, Pani G, Saba A, Pippia P and Cogoli-Greuter M: Space flight affects motility and cytoskeletal structures in human monocyte cell line J-111. Cytoskeleton (Hoboken) 68: 125-137, 2011
15. Hayes JD and McLellan LI: Glutathione and glutathionedependent enzymes represent a co-ordinately regulated defence against oxidative stress. Free Radic Res 31: 273-300, 1999.

16. Chen Y, Johansson E, Fan Y, et al: Early onset senescence occurs when fibroblasts lack the glutamate-cysteine ligase modifier subunit. Free Radic Biol Med 47: 410-418, 2009.

17. Haines DD, Lekli I, Teissier P, Bak I and Tosaki A: Role of haeme oxygenase-1 in resolution of oxidative stress-related pathologies: Focus on cardiovascular, lung, neurologic and kidney disorders. Acta Physiol (Oxf) 204: 487-501, 2012.

18. Lee JC, Kinniry PA, Arguiri E, et al: Dietary curcumin increases antioxidant defenses in lung, ameliorates radiation-induced pulmonary fibrosis, and improves survival in mice. Radiat Res 173: 590-601, 2010.

19. Hayes JD and McMahon M: NRF2 and KEAP1 mutations: permanent activation of an adaptive response in cancer. Trends Biochem Sci 34: 176-188, 2009.

20. Wang J, Zhang J, Bai S, et al: Simulated microgravity promotes cellular senescence via oxidant stress in rat PC12 cells. Neurochem Int 55: 710-716, 2009.

21. Nikawa T, Ishidoh K, Hirasaka K, et al: Skeletal muscle gene expression in space-flown rats. FASEB J 18: 522-524, 2004.

22. Liu Y and Wang E: Transcriptional analysis of normal human fibroblast responses to microgravity stress. Genomics Proteomics Bioinformatics 6: 29-41, 2008.

23. Carson JA, Culberson DE, Thompson RW, Fillmore RA and Zimmer W: Smooth muscle gamma-actin promoter regulation by RhoA and serum response factor signaling. Biochim Biophys Acta 1628: 133-139, 2003

24. Philippar U, Schratt G, Dieterich C, et al: The SRF target gene Fhl2 antagonizes RhoA/MAL-dependent activation of SRF. Mol Cell 16: 867-880, 2004.

25. Beamish JA, He P, Kottke-Marchant K and Marchant RE: Molecular regulation of contractile smooth muscle cell phenotype: implications for vascular tissue engineering. Tissue Eng Part B Rev 16: 467-491, 2010

26. Olson EN and Nordheim A: Linking actin dynamics and gene transcription to drive cellular motile functions. Nat Rev Mol Cell Biol 11: 353-365, 2010.

27. Sun Q, Chen G, Streb JW, et al: Defining the mammalian CArGome. Genome Res 16: 197-207, 2006.

28. Ingber DE: Tensegrity II. How structural networks influence cellular information processing networks. J Cell Sci 116: 1397-1408, 2003.

29. Meloni MA, Galleri G, Pippia P and Cogoli-Greuter M: Cytoskeleton changes and impaired motility of monocytes at modelled low gravity. Protoplasma 229: 243-249, 2006.

30. Servotte S, Zhang Z, Lambert CA, et al: Establishment of stable human fibroblast cell lines constitutively expressing active rho-GTPases. Protoplasma 229: 215-220, 2006.

31. Nichols HL, Zhang N and Wen X: Proteomics and genomics of microgravity. Physiol Genomics 26: 163-171, 2006.

32. Loesberg WA, Walboomers XF, van Loon JJWA and Jansen JA: Simulated microgravity activates MAPK pathways in fibroblasts cultured on microgrooved surface topography. Cell Motil Cytoskeleton 65: 116-129, 2008.

33. Hall A: Rho GTPases and the control of cell behaviour. Biochem Soc Trans 33: 891-895, 2005.

34. Jeggo P: The role of the DNA damage response mechanisms after low-dose radiation exposure and a consideration of potentially sensitive individuals. Radiat Res 174: 825-832, 2010.

35. Jeggo P and Lavin MF: Cellular radiosensitivity: how much better do we understand it? Int J Radiat Biol 85: 1061-1081, 2009.

36. Grimm D, Wise P, Lebert M, Richter P and Baatout S: How and why does the proteome respond to microgravity? Expert Rev Proteomics 8: 13-27, 2011.

37. Gabryś, Greco O, Patel G, Prise KM, Tozer GM and Kanthou C: Radiation effects on the cytoskeleton of endothelial cells and endothelial monolayer permeability. Int J Radiat Oncol Biol Phys 69: 1553-1562, 2007.

38. Rousseau M, Gaugler MH, Rodallec A, Bonnaud S, Paris F and Corre I: Rhoa GTPase regulates radiation-induced alterations in endothelial cell adhesion and migration. Biochem Biophys Res Commun 414: 750-755, 2011.

39. Etienne-Manneville S and Hall A: Rho GTPases in cell biology. Nature 420: 629-635, 2002. 